\title{
Data Analysis for the NASA/Boeing Hybrid Laminar Flow Control Crossflow Experiment
}

\author{
Jenna Eppink ${ }^{*}$ \\ NASA Langley Research Center, Hampton, VA, 23602 \\ Richard Wlezien ${ }^{\dagger}$ \\ Iowa State University, Ames, Iowa, 50011
}

\begin{abstract}
The Hybrid Laminar Flow Control (HLFC) Crossflow Experiment, completed in 1995, generated a large database of boundary layer stability and transition data that was only partially analyzed before data analysis was abruptly ended in the late 1990s. Renewed interest in laminar flow technologies prompted additional data analysis, to integrate all data, including some post-test roughness and porosity measurements. The objective is to gain new insights into the effects of suction on boundary layer stability. A number of challenges were encountered during the data analysis, and their solutions are discussed in detail. They include the effect of the probe vibration, the effect of the time-varying surface temperature on traveling crossflow instabilities, and the effect of the stationary crossflow modes on the approximation of wall location. Despite the low turbulence intensity of the wind tunnel $(0.01$ to $0.02 \%$ ), traveling crossflow disturbances were present in the data, in some cases at amplitudes up to $1 \%$ of the freestream velocity. However, the data suggest that transition was dominated by stationary crossflow. Traveling crossflow results and stationary crossflow data in the presence of suction are compared with linear parabolized stability equations results as a way of testing the quality of the results.
\end{abstract}

\section{Nomenclature}

$C_{p} \quad=$ pressure coefficient

$C_{q} \quad=$ suction coefficient

$\mathrm{c} \quad=$ normal chord

$k=$ stationary mode spanwise wavenumber

Re $\quad=$ Reynolds number

$|\bar{u}|=\quad$ stationary disturbance velocity amplitude

$\bar{u} \quad=$ mean velocity

$u^{\prime} \quad=$ rms disturbance velocity

$U_{\infty} \quad=$ freestream velocity

$x=$ chordwise direction

$y=$ wall-normal direction

$z \quad=$ spanwise direction

$\lambda=$ spanwise wavelength

\section{The Hybrid Laminar Flow Control Experiment}

$\mathrm{T}$ HE NASA/Boeing Hybrid Laminar Flow Control (HLFC) Experiment was an ambitious fundamental transition experiment performed in the NASA Langley 8 -ft. Transonic Pressure Tunnel (TPT). The result was a large and unique set of data. The test was designed over a two-year period, and the testing, which took over a year, was completed in December 1995. Wlezien ${ }^{1}$ gives a historical overview of the test and the then state of the art of technologies that were developed to make the test a successful collaboration between NASA and Boeing. Budget reductions in the late 1990s ended all analysis of the data, but a request was initiated in 2008 to restore the HLFC database to complete the data analysis.

\footnotetext{
* Graduate Student Trainee, Computational AeroSciences Branch, Mail Stop 128, AIAA Student Member

${ }^{\dagger}$ Professor and Vance and Arlene Coffman Endowed Department Chair in Aerospace Engineering, 2271 Howe Hall, Room 1200A, AIAA Fellow 
The purpose of the HLFC experiment was to study the effect of suction on the transition process in swept wing flows. A better understanding of the effects of suction through a perforated skin on crossflow transition in the swept wing boundary layer is needed for HLFC to become a more viable option for laminar flow transport aircraft. The model used in the HLFC experiment was a $3 \mathrm{~m}$ streamwise chord, 35-degree swept wing oriented floor to ceiling in the TPT test section. The model is shown inside of the test section in Fig. 1, where the traverse system is visible towards the back of the wing. The traverse system was moved manually to each of the desired chord locations, and could be controlled remotely to traverse in the spanwise direction. Four interchangeable, identically contoured leading edge panels were used to examine the effects of different suction distributions. Three of the panels were perforated, with hole patterns designed to answer specific questions, and the fourth was a solid panel. The leading edge panel made up the first $20 \%$ of the wing, and needed to be joined smoothly to the midchord region of the wing. This joint location is evident in Fig. 1 as a vertical white line, and this area in particular required a significant amount of work during the experiment to ensure that the flow was not tripped prematurely. For a more detailed description of the experiment, the reader is referred to Choudhari et al. ${ }^{3,4}$

The tests were conducted at a fixed Mach number of 0.24 and chord Reynolds numbers between 5 million and 25 million. The TPT was chosen for its low free-stream turbulence levels $(0.01$ to $0.02 \%)$ in this Reynolds number range. The airfoil shape was designed using CFD and stability prediction codes to obtain crossflow dominated transition. ${ }^{2}$ The airfoil was not symmetric and measurements were only performed on the upper surface, for which the shape and pressure distribution are shown in Fig 2. For more information on the design of the airfoil shape, see Ref. 2. In order to avoid the growth of two-dimensional instabilities, the mid-chord region of the airfoil was cooled by circulating chilled water in channels under the airfoil surface.

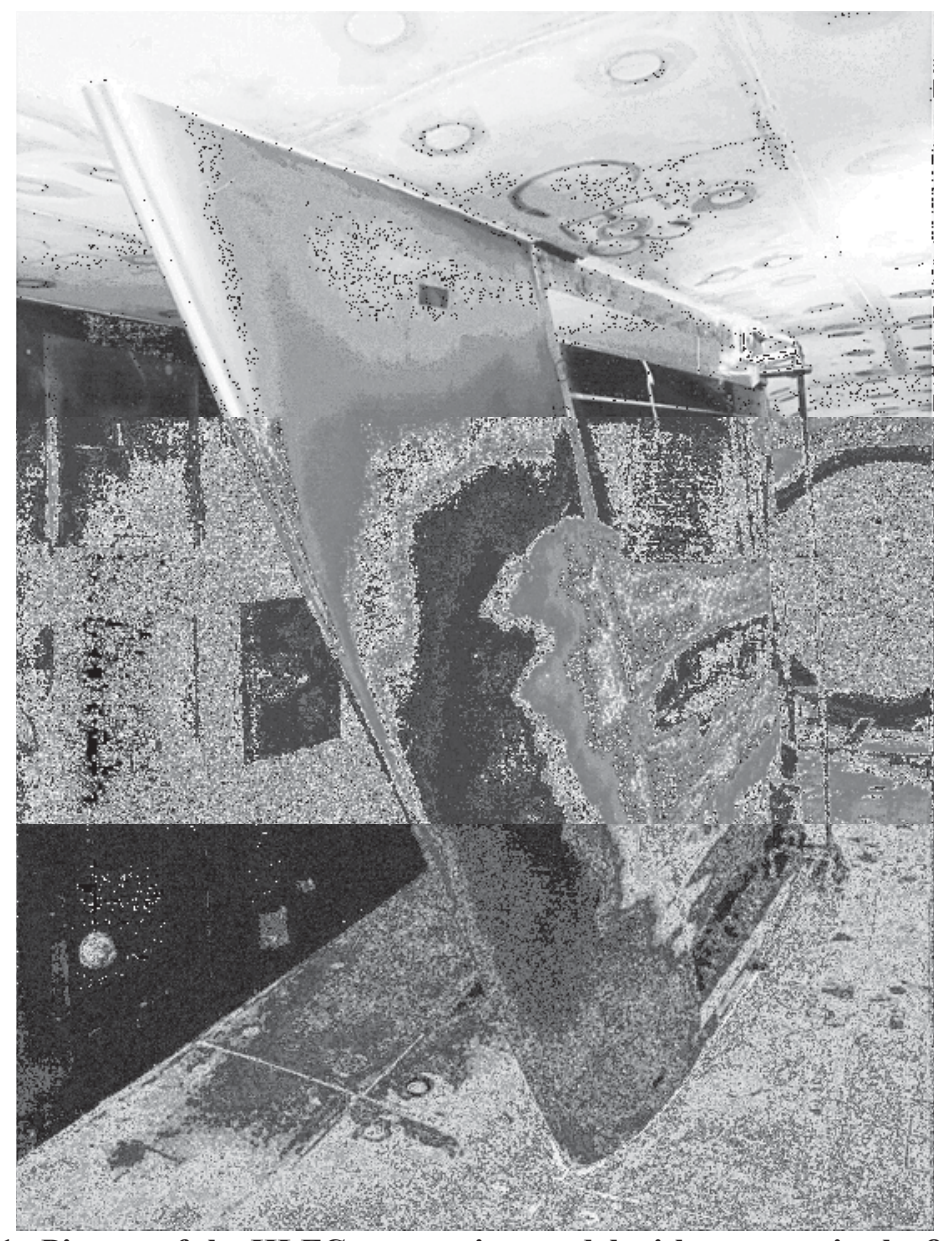

Figure 1. Picture of the HLFC swept wing model with traverse in the $8 \mathrm{ft}$. TPT 

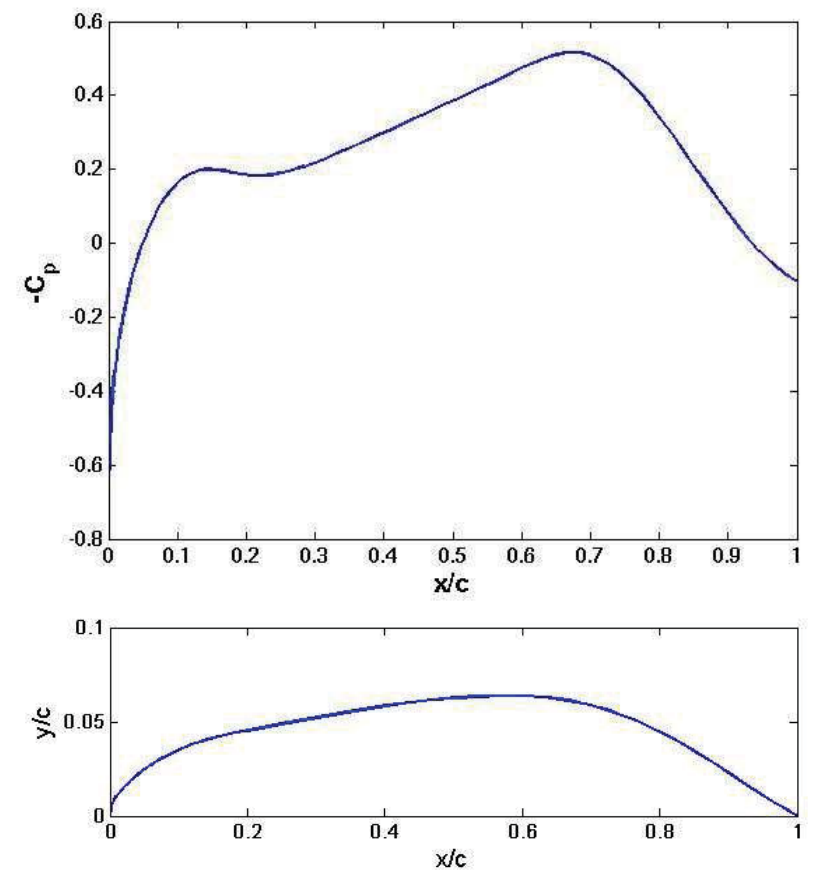

Figure 2. HLFC upper surface model shape and pressure distribution

The suction surfaces on leading edge panels 1 through 3 were divided into 20 individual chambers (called flutes), which spanned the first $20 \%$ chord of the airfoil (Fig. 3). Because the pressure in each flute was controlled separately, many different suction distributions could be tested. The hole size, spacing, and row-to-row hole offset varied between panels. Panel 1 was designed as the most conservative case (i.e. most likely to delay transition), with the smallest sized holes and the finest spacing. Panel 2 was designed to study the detrimental effect of the suction hole pattern on transition. This panel thus had the largest hole size and spacing, and the hole pattern was roughly aligned with the direction of the inviscid streamline and spacing of highly-amplified crossflow modes. Panel 3 was representative of a practical design, with random row-to-row hole offsets and intermediate hole size and spacing. Panel 4 was a solid panel for investigating disturbance growth in the absence of suction.

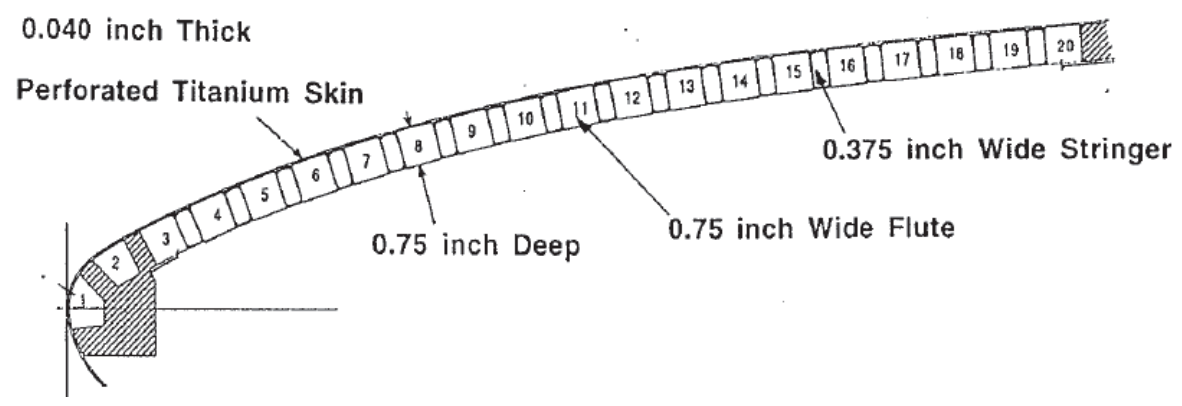

Figure 3. Cross section of leading edge panel showing flute arrangement

The data that was acquired consisted of mean surface pressures, infrared images, and hotwire measurements of steady and time-dependent velocities. A high resolution traversing mechanism was used to acquire boundary layer hotwire scans. Figure 4 shows the coordinate system relative to the wing. Two types of scans were taken: $y-z$ scans and z-scans. The y-z scans consisted of full boundary layer profiles spaced closely together in span in order to gain a full 2D picture of the flowfield. Since the $y-z$ scans proved to be quite time-consuming, a large portion of the data consists of z-scans, which were spanwise scans taken at selected heights above the surface. Dynamic data, sampled at $10 \mathrm{kHz}$, was taken for a limited number of cases of leading edge panel, suction distribution, and chord location. Transition detection was accomplished with surface heat flux measurements using an insulating surface film and an infrared camera. 




Figure 4. Coordinate system for swept wing

Stability analysis was performed for many of the test cases by Choudhari et al. ${ }^{3}$ In this initial look at the data, Nfactor correlations were computed for a number of cases on panels 1,2, and 4; transition locations were determined from the infrared images. The effect of varying the suction distribution was also studied by estimating the transition location from the IR images. Hotwire data was analyzed for leading edge panels 1,2, and 4. None of the dynamic data or the data from leading edge panel 3 was analyzed. More recently a more complete and in-depth look at the HLFC data was initiated, and the data was recovered from encrypted data archives. There were several obstacles to overcome in processing the data and getting it to a point where it could be used. In this paper we discuss some of the techniques used in overcoming those obstacles and some lessons learned from the extended analysis. Some results are also presented for stationary and traveling crossflow on the solid panel, as well as stationary crossflow in the presence of leading edge suction.

\section{Data Analysis}

As is the nature of experimental data, the data from the HLFC experiment are not perfect and need to be corrected relative to known experimental benchmarks before meaningful conclusions can be drawn from the results. In retrospect it turned out to be difficult to ascertain all relevant test conditions, despite efforts to document the test in great detail. Fortunately, many of the missing pieces were eventually discerned, and every possible relevant paramenter was used in the data analysis to make the final results as reliable as possible.

Challenges that needed to be addressed in the data analysis included vibration of the hotwire probe, temperature variation of the cooled airfoil surface, and accurate estimation of the airfoil surface location for analysis of the boundary layer profile scans. In the process of investigating and eliminating these problems, some interesting lessons were learned which have implications for data analysis and future experiments.

\section{A. Probe Vibration}

Vibration of the hotwire probe has always been a concern, and was a major factor in the design of the hotwire traverse system. The very thin laminar boundary layers (on the order of $1 \mathrm{~mm}$ thickness) led to very high velocity gradients which would make the hotwire extremely sensitive to vibration. An accelerometer was located on the hotwire probe head in order to monitor vibration, and spectral coherence with the hotwire signal could be used to deduce vibrational modes. There are several peaks that appear in the power spectral density of the dynamic hotwire data which we believed were due to vibration. However, for several peaks the signal at those frequencies was not coherent with the accelerometer. Thus we loosely categorize the peaks into three classes: probe vibration, travelling crossflow, and other modes.

In order to ascertain traveling crossflow modes, the coherence between the traversing hotwire probe and a fixed probe at a different spanwise location was computed. For a high coherence between the probes, the relative phase 


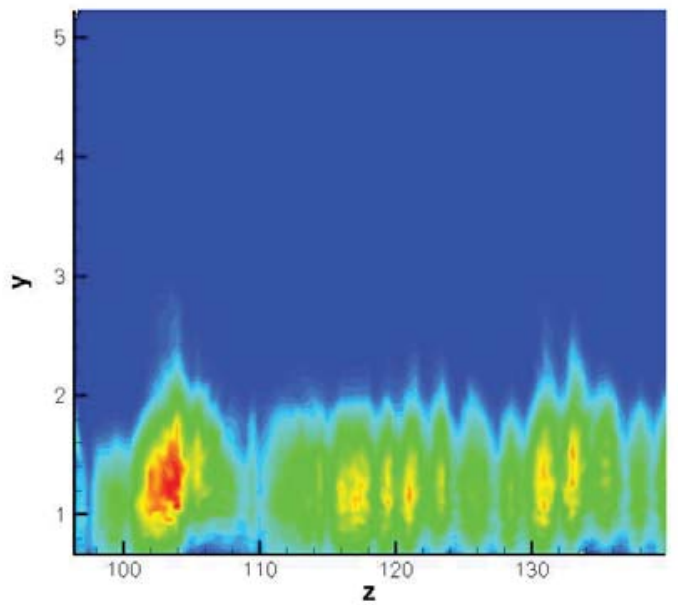

(a) RMS: $\mathrm{f}=\mathbf{2 0 0}-\mathbf{5 0 0} \mathrm{Hz}$

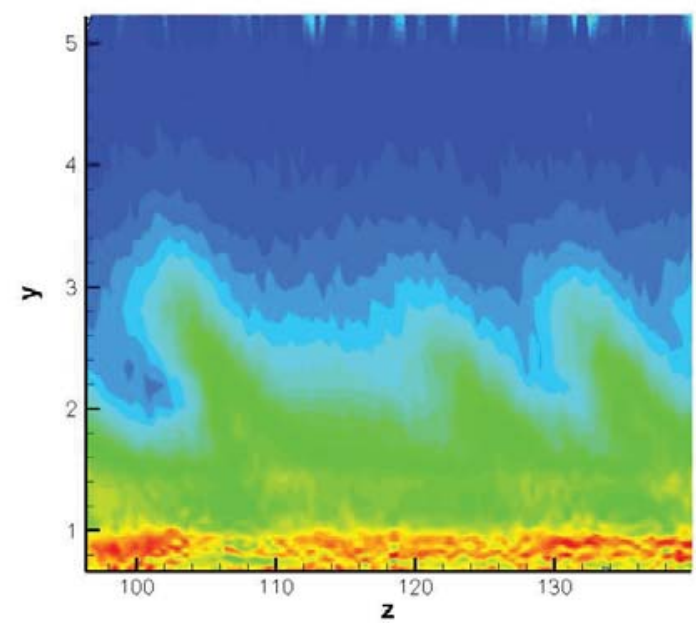

(b) $\mathbf{d u} / \mathbf{d y}$

Figure 5. Plots of y-z scan data from Panel 4: Traveling crossflow mode

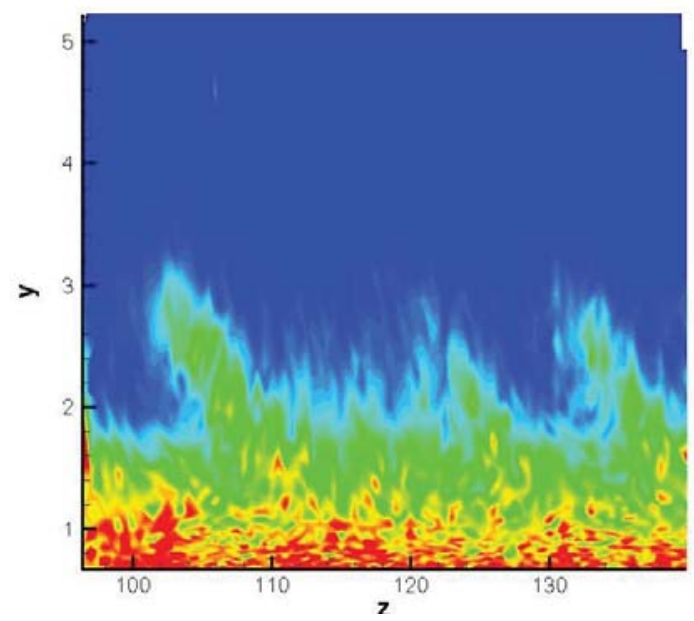

(a) RMS: $\mathbf{f}=30-50 \mathrm{~Hz}$

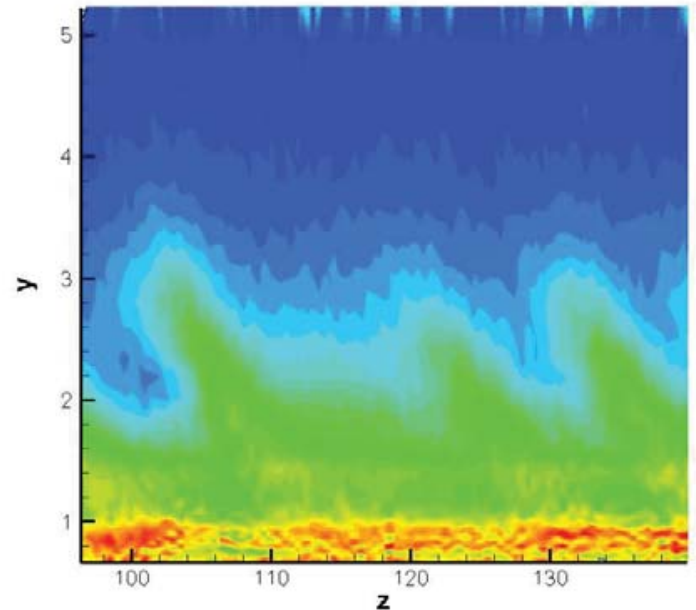

(b) $\mathbf{d u ̄} / \mathbf{d y}$

Figure 6. Plots of y-z scan data from Panel 4: Probe Vibration

could be used to deduce the phase speed of the traveling modes. In the cases where no fixed probe was present, or for identifying the high frequency instabilities that are highly localized and therefore not expected to be coherent with the fixed probe, the fluctuating velocities were qualitatively compared with the mean velocity gradient, $d \bar{u} / d y$. If the sensor is vibrating normal to the wall, the location and magnitude of the fluctuating velocities should correspond to the mean velocity gradient. This is the case for the frequencies that are known to be due to probe vibration.

In these figures, the RMS levels are calculated by first filtering out the frequency content beyond the desired frequency band. Figure 5 shows the comparison of RMS and $d \bar{u} / d y$ over the frequency range known to be traveling crossflow, and, as expected, there is no qualitative alignment with the velocity profile gradient. Figure 6 shows lower frequency content, which is known to be due primarily to vibration of the probe since there is strong coherence with the probe head accelerometer. In this figure, there is strong qualitative alignment with $d \bar{u} / d y$ of the mean velocity profiles.

\section{B. Temperature variation of airfoil surface}

The mid-chord region of the airfoil was water cooled and the surface temperature of the model at this location showed a cyclical variation corresponding to the deadband of the water temperature controller. This cycling had a significant impact on the results for some of the data sets, particularly when the water temperature appeared to be 
cycling over a wider range. Since the data acquisition process for each profile took a fixed time to complete, the temporal wall temperature variation manifests itself as a spanwise variation in temperature. This variation is shown in Fig. 7, in which variation of the water temperature corresponds to the wall temperature.

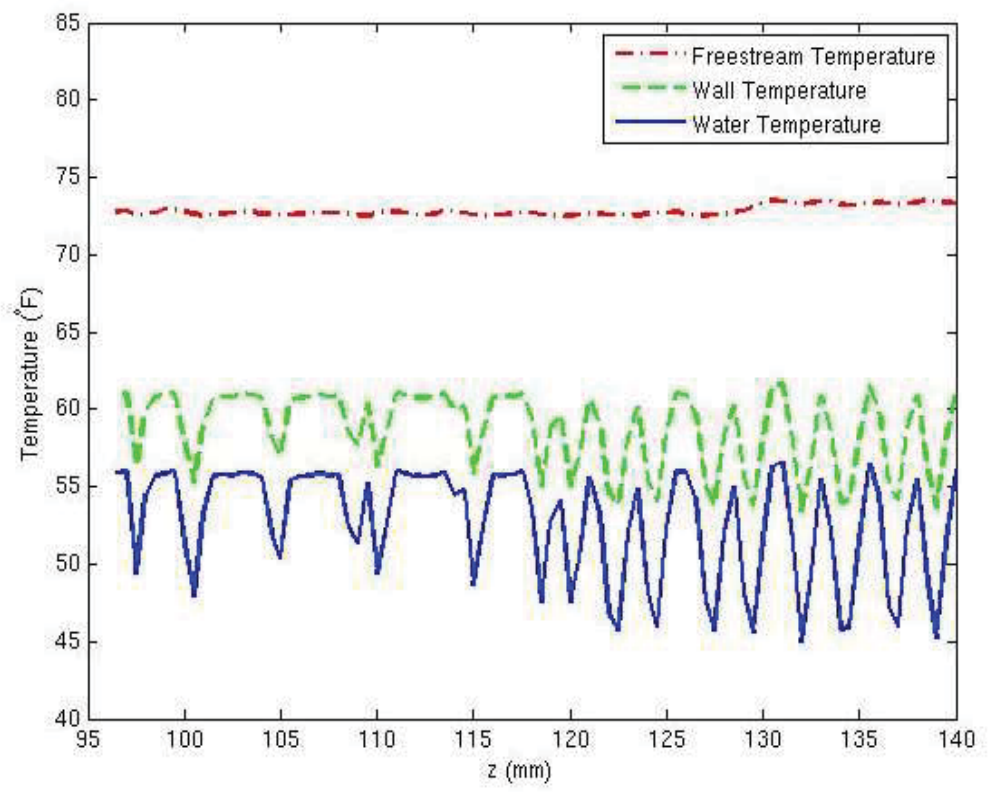

Figure 7. Temperature variation of the water, model surface, and freestream during a $y-z$ scan

This variation in wall temperature influences the temperature profile of the boundary layer. This is important for two reasons. First, it is important to know the local temperature of the air where the hotwire measurements were made so that temperature compensation can be applied to the wire. The temperature compensation for the hotwire measurements was based on an estimate of the temperature profile within the boundary layer. This profile was not measured directly but was calculated based on the local estimate of the velocity profile and the measured freestream and surface temperatures. A second and more complex influence of the temperature variation is the effect it may have had on the instabilities growing inside the boundary layer. It is well-known that wall cooling has a large stabilizing effect on TS disturbances, however, the effect is less important for crossflow disturbances due to the inflectional nature of the instabilities..

Figure 8 is an example of the effect of the temperature variation on the $y-z$ scan data. This plane of data was taken on leading edge panel 4 (the solid panel) at a chord Reynolds number of 5 million, and at a chord location of $\mathrm{x} / \mathrm{c}=0.585$. The temperatures for this case are shown in Fig. 7. The effect of the temperature variation is evident in the 2-D plots of the velocity distributions which have not been temperature compensated. For this case, there was a $350 \mathrm{~Hz}$ traveling crossflow mode present, so the $1 \mathrm{kHz}$ sampling rate of the $\mathrm{y}-\mathrm{z}$ scan data was able to capture it. Therefore, we are also able to look at a 2D map of the RMS amplitude of the traveling crossflow disturbances (Fig. $8 \mathrm{~b})$ as they are modulated by the stationary mode. The temperature compensated mean and RMS velocities are plotted in Fig. 9. The short wavelength variations in both the RMS and the mean velocity plots in Fig. 8 correspond to the variation of the surface temperature (and therefore the water temperature). This is highlighted in Fig. 10a, which shows the high-pass filtered mean and RMS velocities at a constant y-location, as well as the water temperature.

The temperature compensated plot of the mean data (Fig. 9) shows that proper temperature compensation removes most of the variations that correspond to the water temperature cycling. This is also illustrated in Fig. 10b, where the high pass filtered spanwise variations in mean flow before and after temperature compensation are plotted. While there is still some variation evident after the temperature compensation is applied, it is not nearly as significant as before. The remaining variations may be due to uncertainties in the temperature measurements, temperature profile estimation, or in the application of the hotwire temperature compensation. However, the RMS plot looks very similar to the corresponding plot of the uncompensated data. It appears that the effect of the temperature difference on the hotwire does not correspond to the variations in the amplitude of the traveling disturbances. We hypothesized that the amplitude of the traveling crossflow was actually influenced by the temperature variations, and we investigated this behavior by comparing the experimental data with computations. 


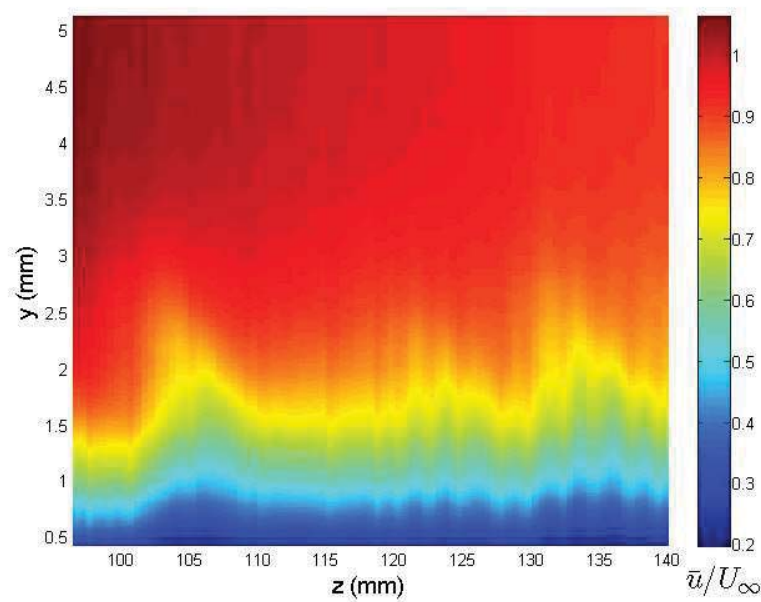

(a) Mean Velocity

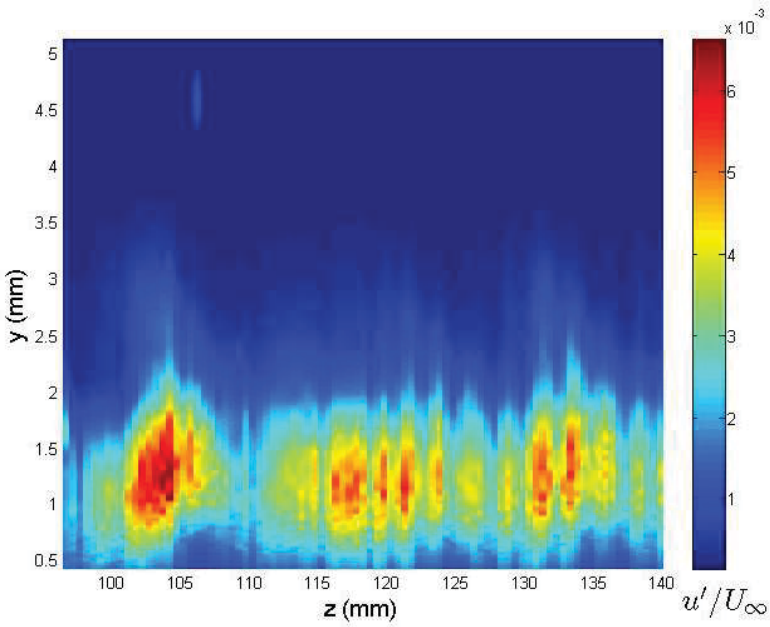

(b) RMS Velocity

Figure 8: Plots of mean velocity and RMS velocity $(\mathrm{f}=\mathbf{2 0 0}$ to $500 \mathrm{~Hz})$ with no temperature compensation applied.

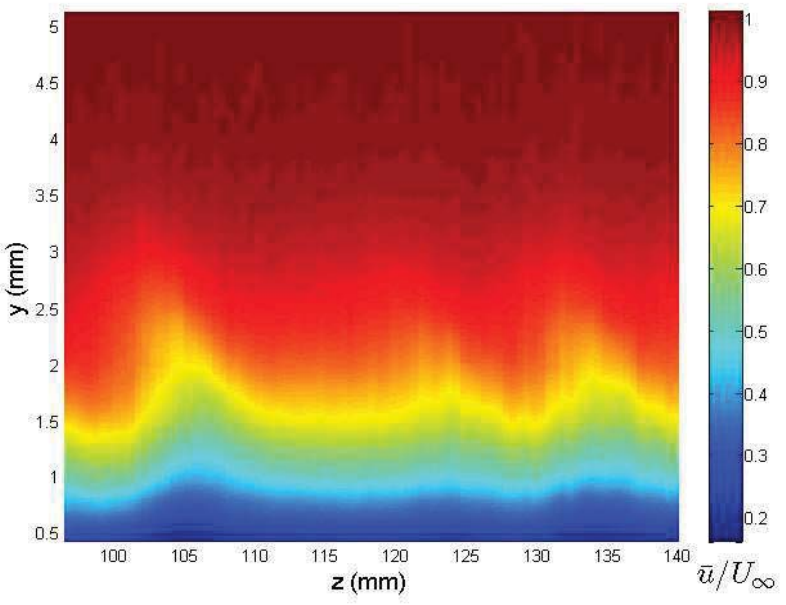

(a) Mean Velocity

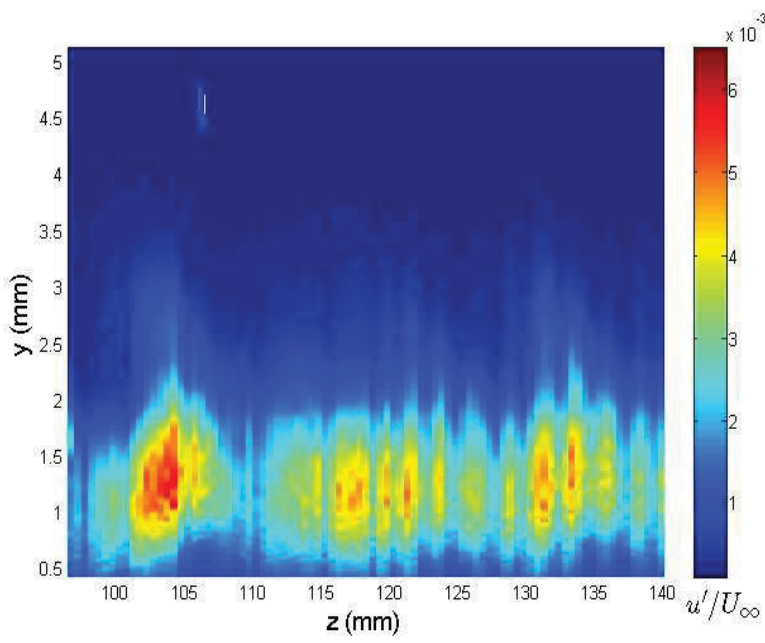

(b) RMS Velocity

Figure 9: Plots of mean velocity and RMS velocity $(\mathrm{f}=\mathbf{2 0 0}$ to $500 \mathrm{~Hz}$ ) with temperature compensation applied.

The data from $\mathrm{z}=120$ to $140 \mathrm{~mm}$ was first divided into regions of high $\left(\geq 58^{\circ} \mathrm{F}\right)$ and low $\left(\leq 56^{\circ} \mathrm{F}\right)$ wall temperature, and then the average traveling mode shape was calculated for the low and high temperature sets of profiles. The result is plotted in Fig. 11. The high temperature amplitude is about $23 \%$ larger than the low temperature mode. This result was then compared to the linear PSE results computed using LASTRAC. ${ }^{5}$ The Nfactors were computed for a traveling mode with $\mathrm{f}=350 \mathrm{~Hz}$ and $\lambda=22 \mathrm{~mm}$, which was the frequency and spanwise wavelength of the traveling mode that was present in the experimental data. The chordwise temperature profile was estimated by Choudhar ${ }^{3,4}$ by applying a smooth fit to surface temperature measurements made during the experiment. This temperature profile is shown in Fig. 12. For the low temperature case, this same profile was used but was shifted down by $7^{\circ} \mathrm{F}$.

The $\mathrm{N}$-factor plot (Fig. 13) does show an increase in $\mathrm{N}$-factor for the higher temperature case, which is consistent with the experimental data (Fig. 10). The difference in $\mathrm{N}$-factors at $\mathrm{x} / \mathrm{c}=0.585$ is 0.32 , which corresponds to an amplitude ratio of 1.377 , assuming the initial amplitude does not change. The actual amplitude ratio of the high and low temperature modes in Fig. 12 is 1.2979. Despite the fact that the difference in N-factor due to the change in temperature appears quite small, it is in fact more than sufficient to produce the measured amplitude 


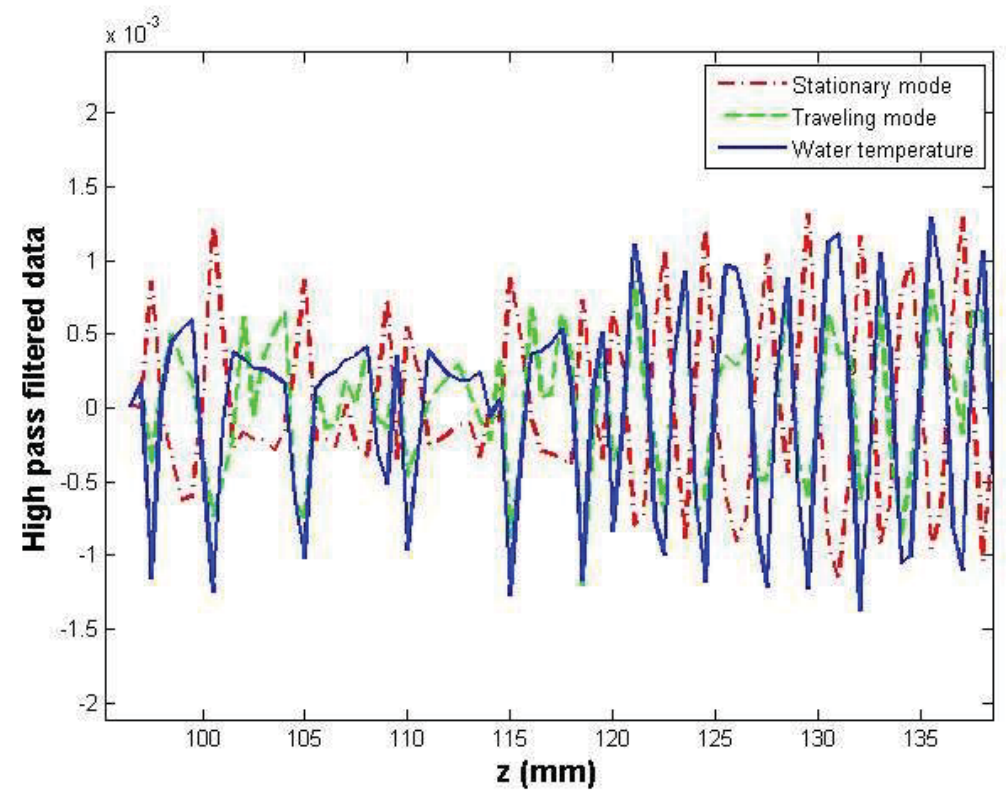

(a) Plot of variations in the mean flow (Stationary mode), RMS velocity (Traveling mode), and water temperature

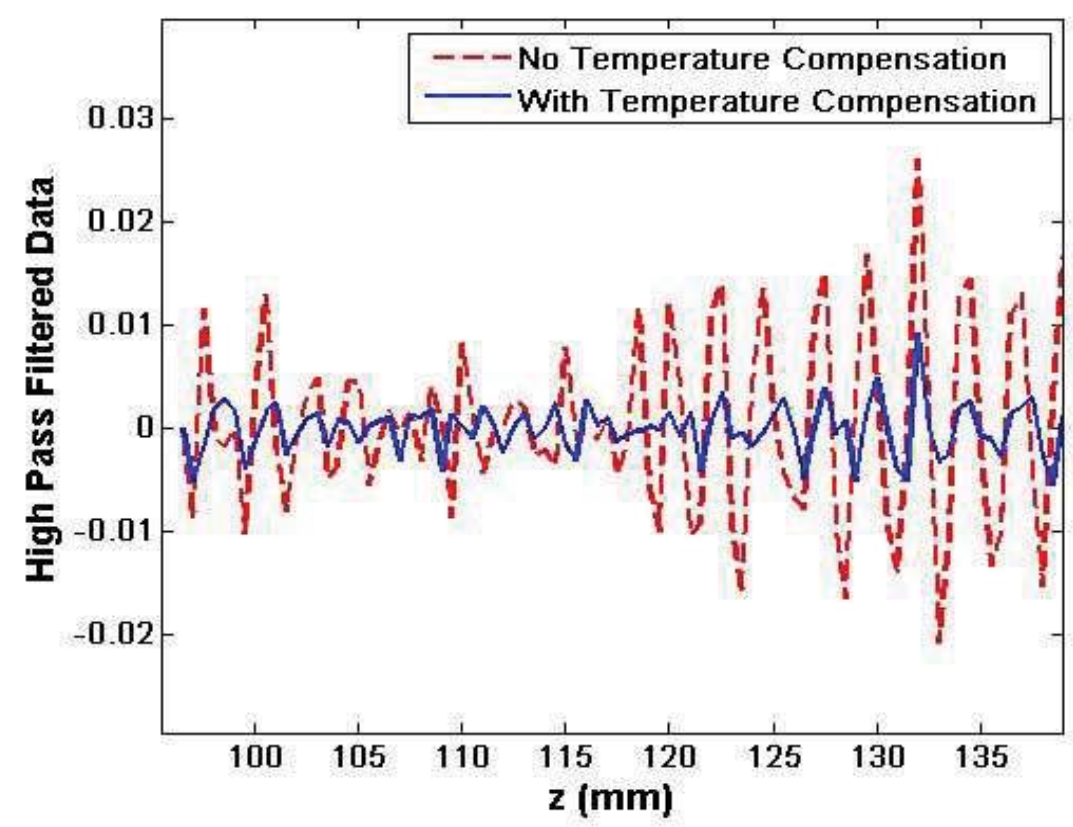

(b) Plot of variation in the mean flow before and after temperature compensation was applied

Figure 10. High wavenumber variations in mean flow and RMS Velocity (at a constant y-location), and water temperature. (In (a) the values have been scaled arbitrarily for sake of comparison) 


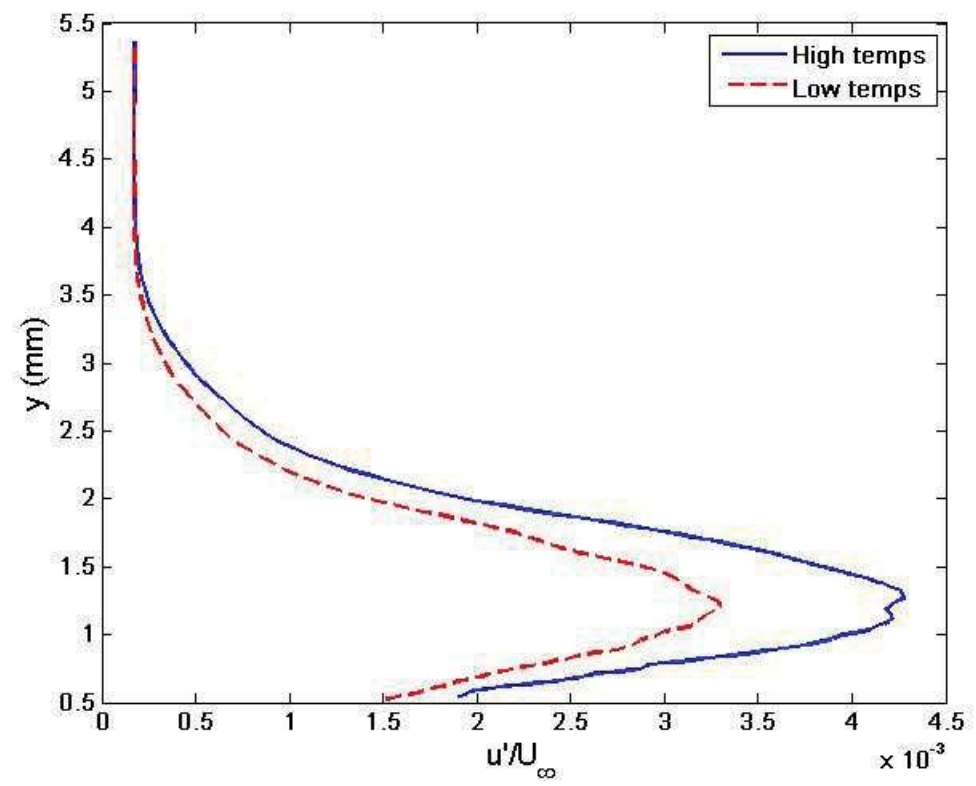

Figure 11. Traveling crossflow mode at high and low temperatures

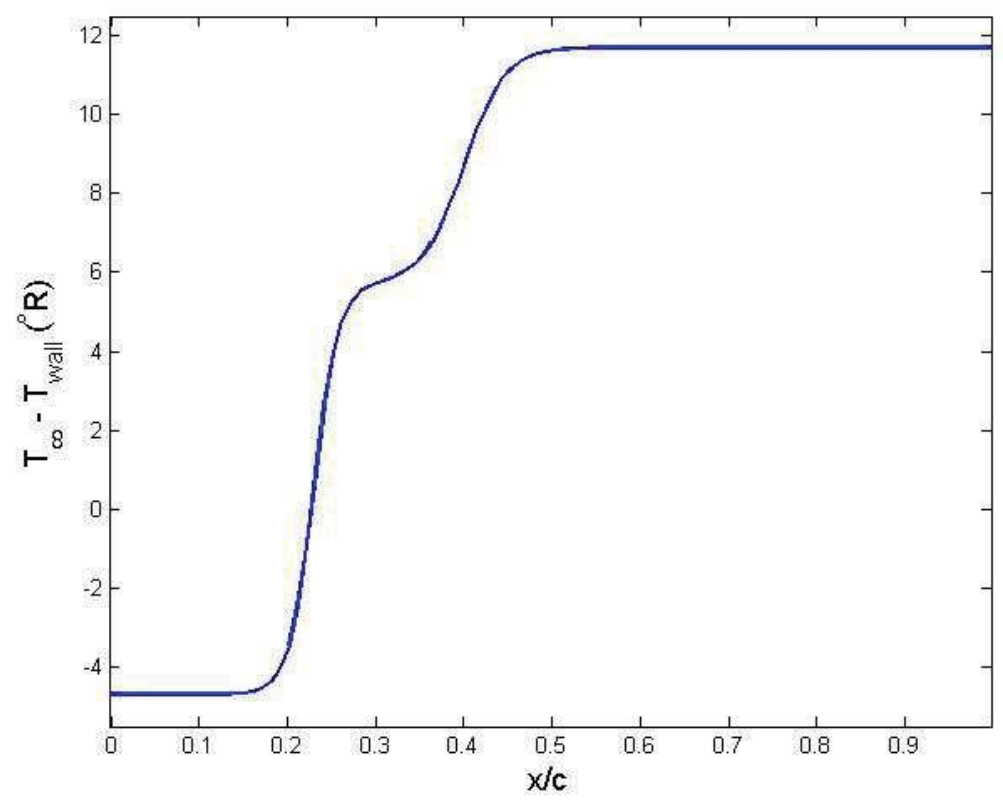

Figure 12. Chordwise wall temperature distribution 


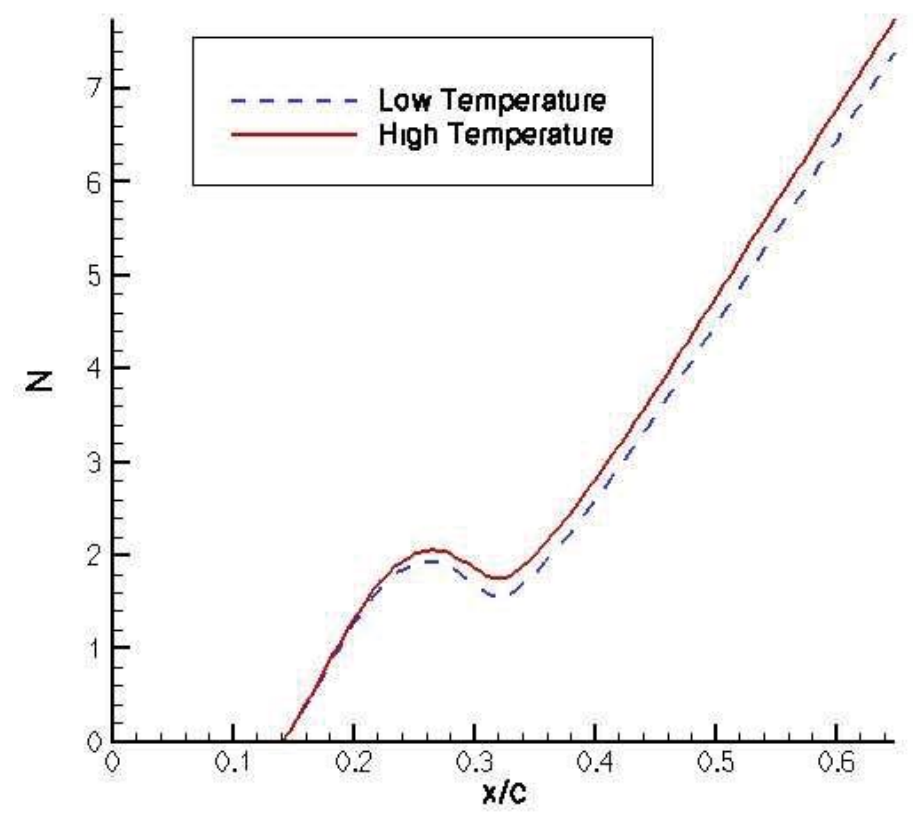

Figure 13. Linear PSE traveling crossflow N-factors computed using LASTRAC

difference that is seen in the experimental data. We show it to be quite plausible that the variations seen in the RMS values may be due to a change in the amplitude of the traveling crossflow.

This result is significant because it indicates that one needs to be particularly careful when carrying out experiments where traveling disturbances could affect the results. One such example is in temperature sensitive paint (TSP) experiments on swept wings, in which a temperature step is introduced by modifying the freestream temperature (by injecting more or less nitrogen in cryogenic tunnels). Fey ${ }^{6}$ observed a difference in the results when a negative temperature step was used instead of a positive temperature step. The transition location for the negative temperature step (where the model was warmer than the freestream) moved upstream relative to the corresponding positive temperature step cases. This result was for a fairly large temperature difference $\left(43^{\circ} \mathrm{F}\right)$. As the temperature steps were decreased, the difference between the positive and negative temperature changes was no longer visible in the images. Based on the present results, this difference in transition location could very well have been due to the destabilizing effect of warmer model temperature on the traveling crossflow modes.

Linear PSE results were also computed for the stationary crossflow mode. Similar to traveling crossflow, the stationary crossflow mode was predicted to be stabilized by a decrease in the wall temperature. However, the experimental data show an opposite trend (Fig. 10), and therefore the variations seen in the stationary crossflow are probably due to the temperature effect on the hotwire, as demonstrated in Fig. 9. The effect of the temperature difference on the stationary crossflow amplitude was predicted to be much smaller than the effect on the traveling crossflow (the difference in $N$-factor at $\mathrm{x} / \mathrm{c}=0.585$ was only 0.08 ).

\section{Wall Location}

When using a hotwire to characterize transitional boundary layers, it is typically not possible to measure velocity all the way down to the wall. A wall location algorithm must be used to find the location of the wall relative to the sensor. The traditional method of locating the wall consists of a curve fit (either linear or full profile) to the individual profile. In a linear fit, the lower portion of the velocity profile is extrapolated linearly to the wall. A full profile fit utilizes the entire measured profile and some assumed profile shape. Both linear and global fitting methods were utilized in the initial analysis of the HLFC data, ${ }^{3,4}$ and both proved to give similar results. However, when the wall location is plotted in $\mathrm{z}$, a regular variation is evident which corresponds to the dominant crossflow wavelength. Figure 14a shows the wall location that results when a linear fit is applied to the lower portion of the boundary layer profiles for an entire plane of data (labeled "Linear Fit Wall Location" in the plot). The wall location in this plot is negative because the wall normal measurements were made in reference to a location above the airfoil surface. This profile can be compared with Fig.14b, which shows the mean flow distribution. There is a 
clear spanwise variation in the wall location that corresponds to the variations in the mean flow contours. This is not surprising, considering that the individual profiles are distorted by the strong crossflow mode(s).

Surface roughness measurements were performed on leading edge panel 4 after the completion of the test. According to these measurements, the peak to peak roughness of the model was on the order of $0.01 \mathrm{~mm}$, whereas the variation in the calculated wall location (as in Fig. 14a) is on the order of $0.5 \mathrm{~mm}$. When y-z scans were made at the same location, but under different conditions, the spanwise wavelength of the dominant stationary disturbance was different. If the variations were real, then the wall location would not change from case to case. However, in every case, the spanwise variation that occurred in the calculated wall location corresponded to the wavelength of the stationary crossflow pattern. Both of these observations indicate that the variations in wall location were not actually indicative of model surface roughness or waviness.

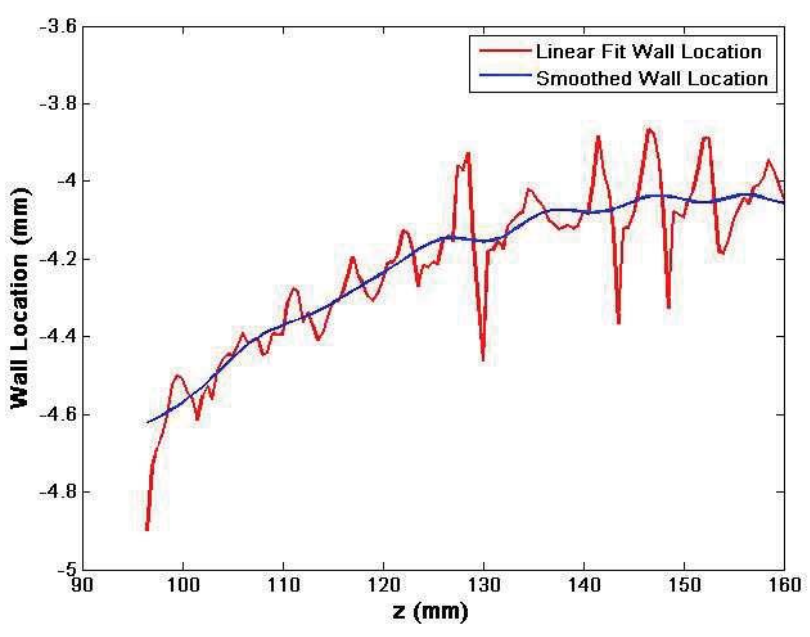

(a) Wall location vs. $z$

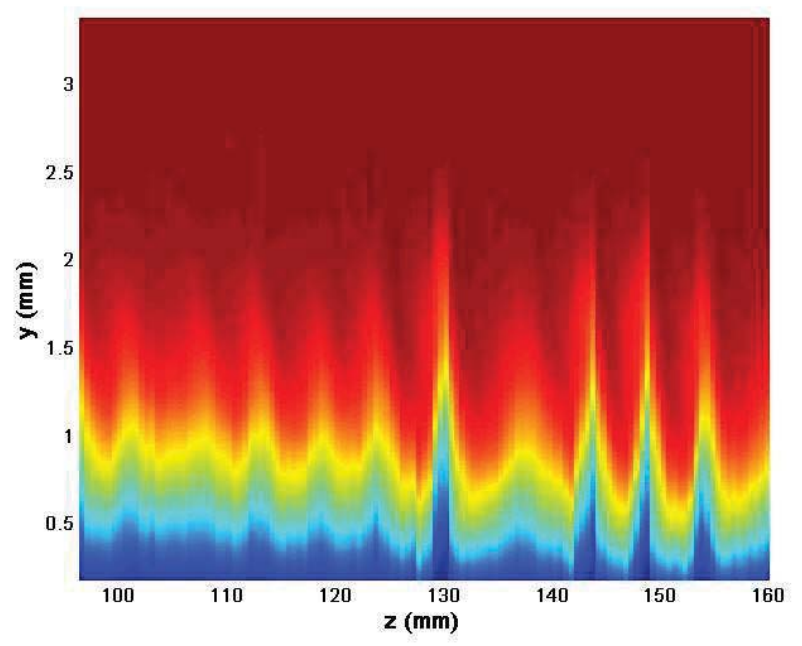

(b) Mean flow

Figure 14. (a) Wall locations calculated using linear fit and the smoothed wall technique. (b) Mean flow for this plane using the linear fit wall location. Z-scale values are $\bar{u} / U_{\infty}$.
The solution that seems most reasonable is to filter out the variations in wall location using a spatial low pass filter, so that only the larger wavelength variations remain. Low wavenumber changes could be due to a slightly-misaligned traverse, or actual waviness in the airfoil surface. The profile to profile variations (on the order of $0.5 \mathrm{~mm}$ spacing) are also assumed to be unreal, so these are filtered out as well. These variations could be due to the inherent uncertainties in the linear fit wall finding routine or near wall effects on the hotwire data.

An example of the smoothed wall location that results from this process is plotted in Fig. 14a. The result is smoother velocity contours (mostly from filtering out the profile to profile variations), and changes in crossflow mode shape and amplitude. Fig. 15 shows the difference between the stationary crossflow mode shape using the wall location calculated from the linear fit, and the mode calculated using the smoothed wall for one case. In this case, the smoothed wall location results in a $25 \%$ reduction in amplitude and a crossflow mode shape where the maximum has moved closer to the wall. Using the smooth wall technique does not reduce the amplitude of the crossflow mode in every case, but in every case there is at least a $10 \%$ difference in amplitude; in some cases this difference is as high as $40 \%$. In comparisons with theoretical mode shapes computed using linear PSE, shown in Fig. 16, the smooth wall results show slightly better agreement for most cases, though in some cases it is impossible to determine which shape is closer to the theoretical shape. Despite the fact that these two datasets were taken at different locations and with different dominant mode wavelengths, the effects of the smooth wall technique are quite similar. In both cases, the mode shape resulting from the smooth wall technique shows much better agreement with theory near the wall than does the mode shape resulting from the linear fit method. The results that are presented hereafter were analyzed using the smooth wall technique. 


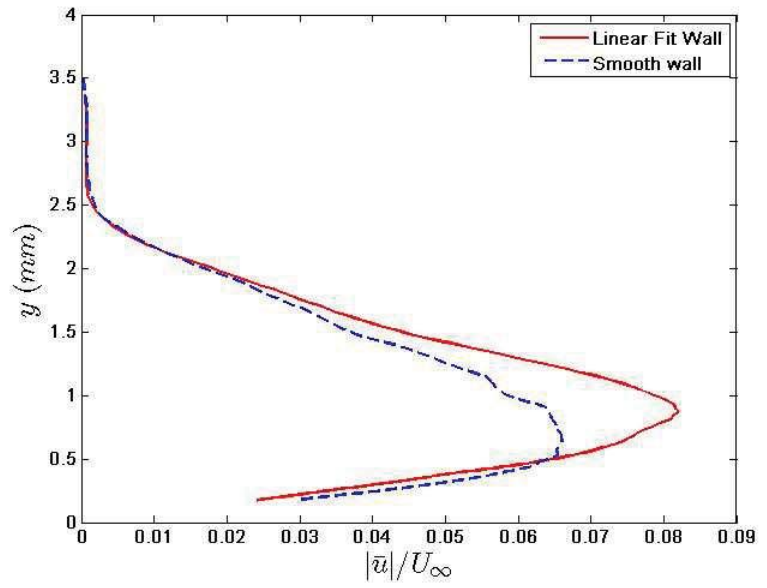

Figure 15. Comparison of crossflow modes with the linear fit calculated wall and the smoothed wall

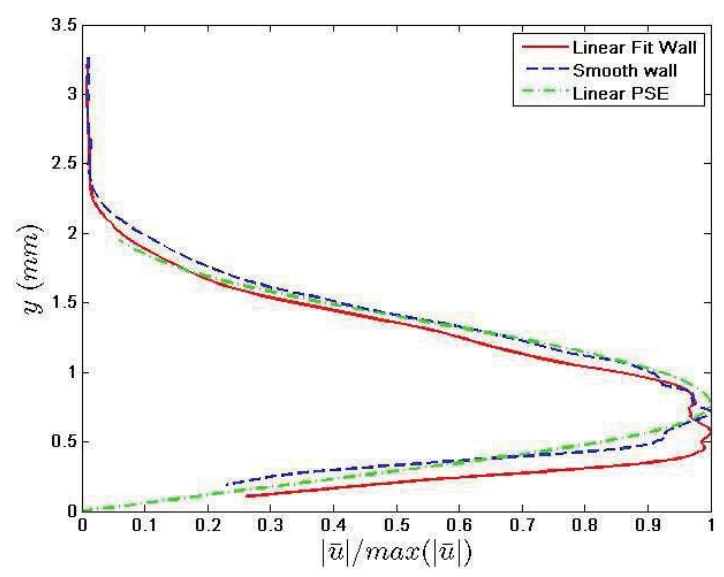

(a) $4 \mathrm{~mm}$ mode $(\mathrm{x} / \mathrm{c}=0.257, \operatorname{Re} / \mathrm{ft}=1 \mathrm{M})$

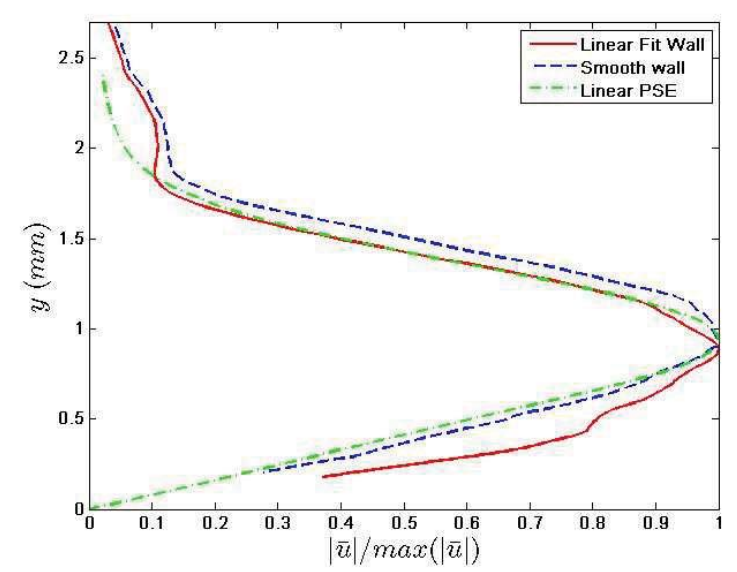

(b) $10 \mathrm{~mm}$ mode $(\mathrm{x} / \mathrm{c}=0.437, \operatorname{Re} / \mathrm{ft}=1 \mathrm{M})$

Figure 16. Comparison of experimental crossflow modes with numerical mode shapes

\section{Traveling Crossflow}

Traveling crossflow on swept wing models has not been very thoroughly studied in the past, particularly for cases of natural transition. Dehyle ${ }^{7}$ has studied natural transition on swept flat plates, and White and Saric ${ }^{8}$ have studied forced crossflow transition on swept wings with discrete roughness elements. Dagenhart and Saric ${ }^{9}$ found traveling crossflow in a swept wing model under natural transition, however, the traveling modes were not studied in great detail. For the HLFC experiment, dynamic data was taken for a limited number of cases and locations. These data sets consisted of roughly 8 to 10 boundary layer profiles spaced a few millimeters apart in span. The dynamic data sets were sampled at $10 \mathrm{kHz}$, as opposed to the $1 \mathrm{kHz}$ sampling rate of the stationary data. These data sets were usually only taken at one chord location for each flow condition and suction configuration, and this location was usually far back on the airfoil, near the transition location. While dynamic data was being acquired, a fixed hotwire probe located at a fixed location within the boundary layer was simultaneously acquiring data. This data is used to determine the coherence with the traversing probe and the phase speed of the traveling modes.

An interesting outcome of the HLFC experiment was the appearance of traveling crossflow disturbances at significant amplitudes (up to $1 \%$ of the freestream velocity). While the traveling disturbance amplitude was too low to have a significant effect on the transition process, it is still a surprising outcome due to the low turbulence intensity of the tunnel at the flow conditions run in the experiment $(0.01$ to $0.02 \%) .{ }^{10}$ At such a low turbulence intensity, traveling crossflow disturbances are not expected to be present at significant amplitudes. Even more interesting, the cooling of the midchord region of the airfoil was expected to have a stabilizing effect on traveling disturbances, so without the cooling of the airfoil surface, the traveling crossflow disturbances probably would have had even larger amplitudes. 
The phase speed and mode shape of the traveling disturbance was computed for the Panel 4 case discussed earlier, at a chord Reynolds number of 5 million, and chord location of $x / c=0.585$. The dynamic results from the $y-z$ scan for this case are plotted in Fig. 9b. Spanwise modulation of the traveling crossflow due to the strong stationary crossflow mode is evident in this plot. The y-z scans were not intended to provide data on traveling disturbances, so there was no fixed probe present for these data sets. Hence, we must use the dynamic data to obtain phase speed information. The dynamic data for this case consisted of 7 profiles taken from $z=96$ to $119 \mathrm{~mm}$. The amplitude of the $350 \mathrm{~Hz}$ traveling crossflow mode, which was the dominant mode in the experimental data, was around $1 \%$ of the freestream velocity. At a frequency of $350 \mathrm{~Hz}$, there was strong coherence with the fixed probe, and therefore a phase shift could be computed. The phase shift at each location was computed and this data is plotted in Fig. 17. From the slope of the line, the spanwise phase speed was determined to be $8 \mathrm{~m} / \mathrm{s}$, which corresponds to roughly $10 \%$ of the freestream velocity. Unfortunately, there was not enough information to compute wave angle. The spanwise phase speed was used to calculate the spanwise wavelength of the traveling crossflow mode, which was found to be $22 \mathrm{~mm}$. The linear PSE results for this case predict that the most amplified mode will have a frequency of $250 \mathrm{~Hz}$ and a wavelength of $18 \mathrm{~mm}$ at this chord location, which is different from the experimental mode that was measured. Linear PSE predicts a spanwise phase speed of $4.5 \mathrm{~m} / \mathrm{s}$, which is roughly $5 \%$ of the freestream velocity. The average mode shape of the measured traveling mode $(\mathrm{f}=350 \mathrm{~Hz}, \lambda=22 \mathrm{~mm})$ is plotted in Fig. 18a along with the mode shape calculated using LASTRAC. This plot shows nearly perfect agreement between the experimental and theoretical traveling crossflow mode shapes.

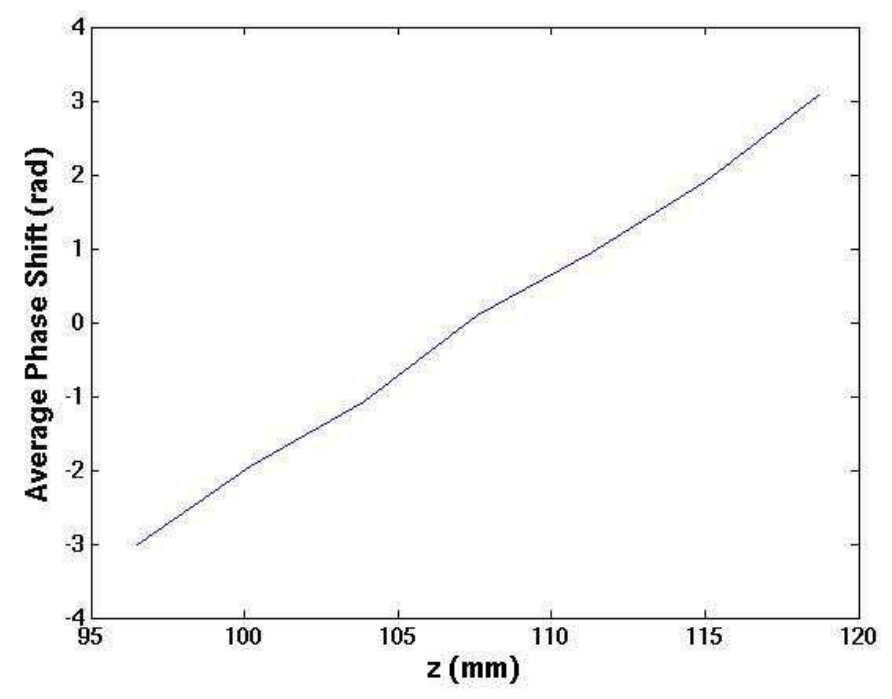

Figure 17. Phase shift vs. $\mathrm{z}$ for $\mathrm{Re}=5 \mathrm{M}, \mathrm{x} / \mathrm{c}=\mathbf{0 . 5 8 5}$ on Panel 4

The dominant stationary crossflow mode at this location had a wavelength of around $12 \mathrm{~mm}$, and an amplitude of $3 \%$ of the freestream velocity. Linear PSE predicts that the most amplified mode will be around $12 \mathrm{~mm}$ as well. The mode shape is compared with linear PSE results in Fig. 18b. The shapes agree fairly well, but there appears to be a vertical shift up in the experimental mode relative to the theoretical mode. There is also some disagreement in shape near the wall. This difference may be due to uncertainties in the determination of the wall location.

Traveling crossflow was present in most of the cases studied, with or without suction. In the cases studied on Panel 2, for which stationary crossflow results are presented in the following section, dynamic data were taken at the furthest downstream measurement location: $\mathrm{x} / \mathrm{c}=0.437$. For these cases, the traveling crossflow amplitudes were around $0.3-0.5 \%$ of the freestream velocity. Mode shapes match linear PSE results fairly well, although there appear to be vertical shifts in some cases, similar to Fig. 18b. As in the Panel 4 case discussed, the amplitude of the dominant stationary crossflow mode in these cases was around $4 \%$ of the freestream velocity. The experimentally measured spanwise phase speeds in these cases were between 10 and $13 \%$ of the free of the freestream velocity. The phase speed values measured in this experiment are similar to the results of Takagi and Itoh ${ }^{11}$, who measured a phase speed of $17 \%$ of the freestream velocity. However, as in the Panel 4 case discussed previously, the linear PSE results give a phase speed of around 5\% for the most amplified mode. 


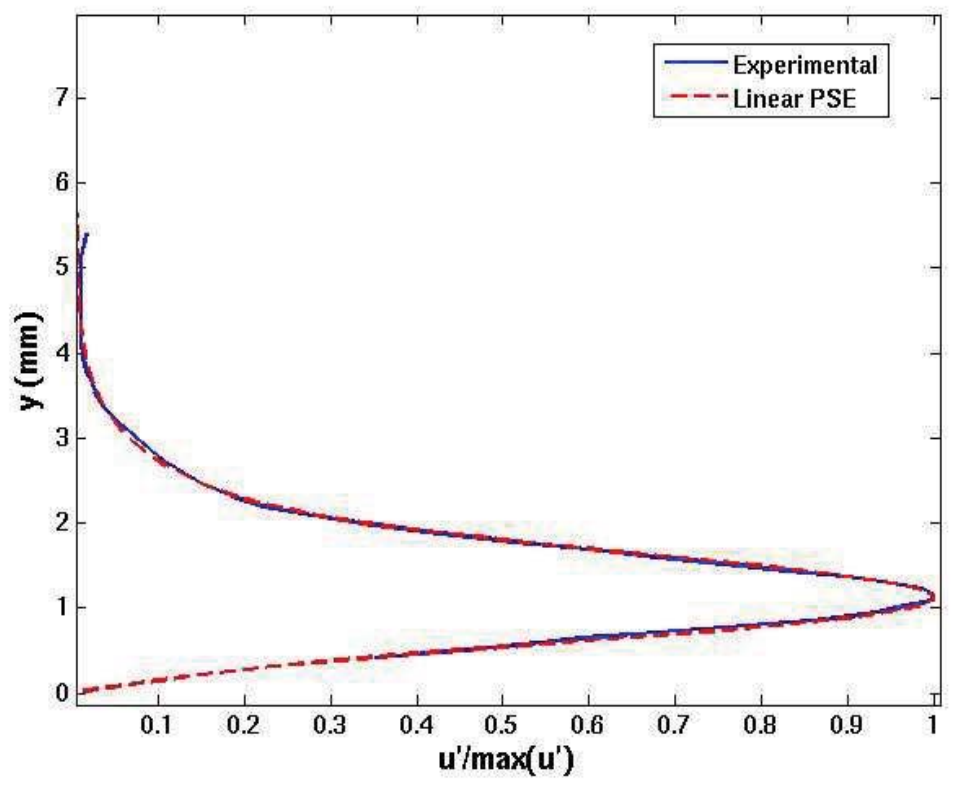

(a) Traveling crossflow mode (Experimental: $f=300$ to $400 \mathrm{~Hz}$, Numerical: $f=350 \mathrm{~Hz}$ )

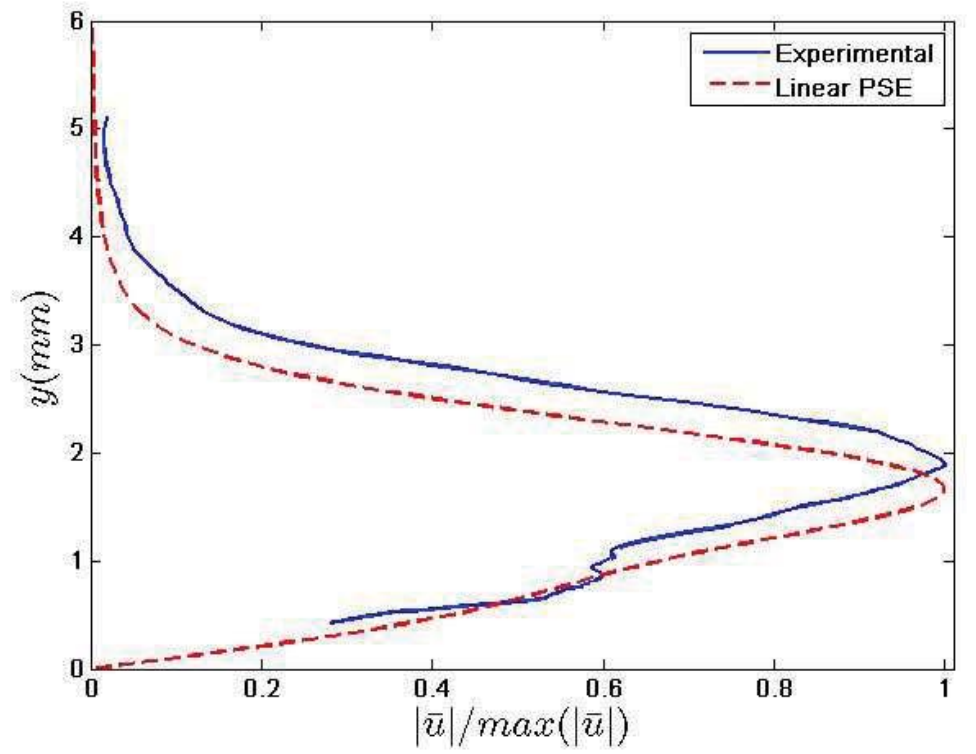

(b) Stationary crossflow mode (Experimental: $\mathrm{k}=0.05$ to $0.1 \mathrm{~mm}^{-1}$, Numerical: $\mathrm{k}=0.083 \mathrm{~mm}^{-1}$

Figure 18. Comparison of disturbance mode shapes with linear PSE for $\mathrm{Re}=5 \mathrm{M}, \mathrm{x} / \mathrm{c}=\mathbf{0 . 5 8 5}$ on Panel 4 


\section{Effect of Varying Suction Distribution}

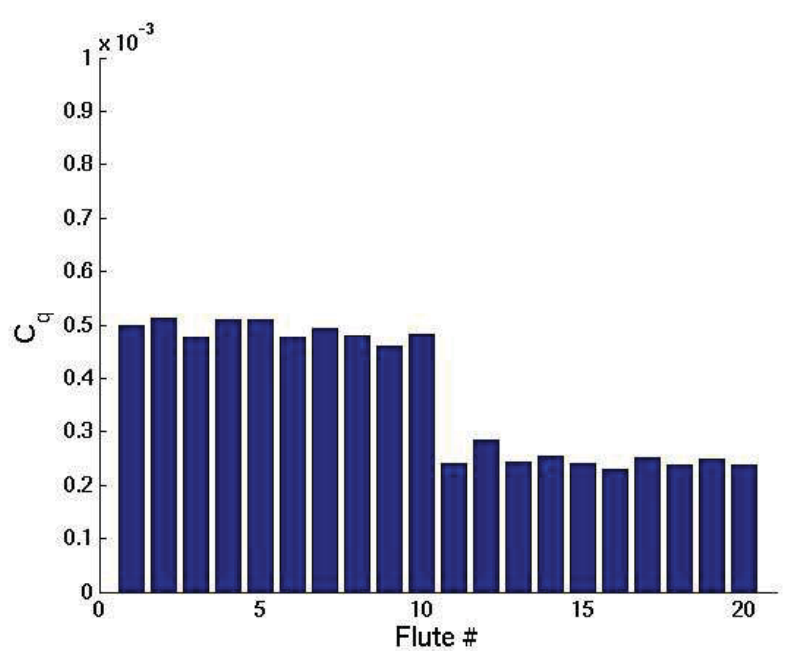

(a) Suction Distribution 5

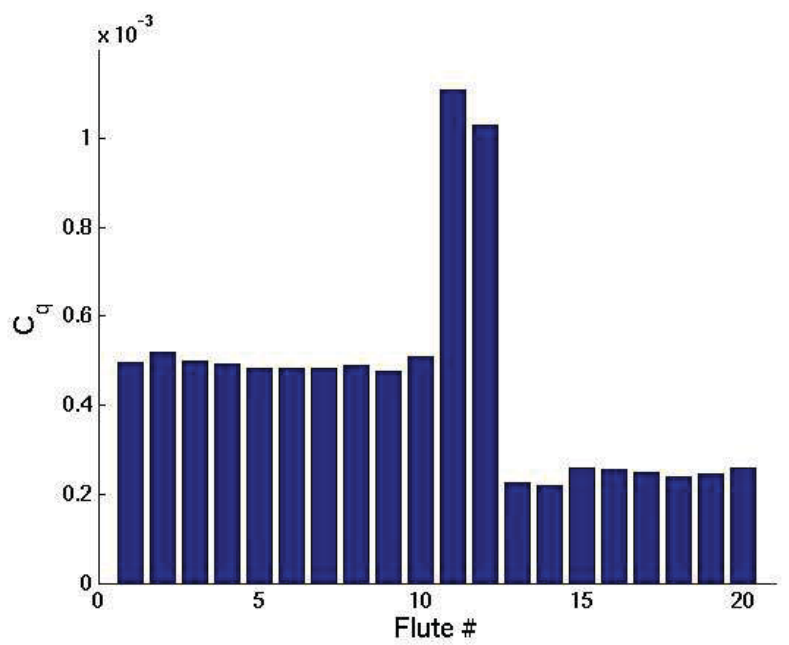

(b) Suction Distribution 108

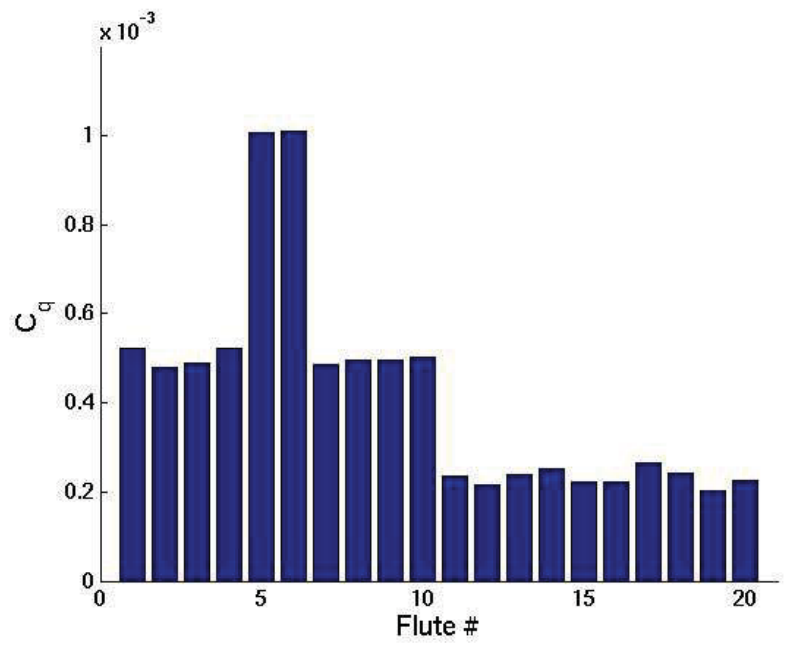

(c) Suction Distribution 110

Results from several different suction distributions on Panel 2 are discussed and compared with numerical results. The effect of suction on transition in swept wing flows has been studied both experimentally and numerically since it was first used as a means of delaying transition. The experiments that have been performed have mainly studied the effect of suction distribution on transition location, ${ }^{12,13}$ and the effect of hole size and spacing on transition location. ${ }^{14}$ However, the NASA/Boeing HLFC dataset is unique in that it contains detailed measurements of crossflow instabilities for varying suction distributions. This data can be very useful for code calibration and can help provide an increased understanding of the effect of suction on crossflow instabilities.

A significant number of $y-z$ scans were taken on leading edge panel 2 (which was the panel with the largest hole size and spacing), therefore more information is available from these results than from other cases where only z-scans were taken. This is also an interesting case because this panel was designed to study the detrimental effect of suction hole size and spacing on crossflow instability growth.

Three slightly different suction distributions are discussed below, for which y-z scans were taken at three chordwise locations $(\mathrm{x} / \mathrm{c}=0.157$, 0.257 , and 0.437). Unfortunately, the exact transition locations for these cases are unknown, due to the fact that there were no IR images taken when the hotwire data was acquired. The three suction distributions tested include the baseline suction distribution (labeled Suction Distribution 5) and two variations on the baseline in which the suction through two adjacent flutes was increased above the baseline level (Suction Distributions 108 and 110, see Fig. 19).

The dominant mode near the leading edge $(\lambda \sim 3-4 \mathrm{~mm})$ corresponds to the suction hole spacing on the panel. This was expected to happen on this panel, since the hole spacing was designed to correspond to an unstable mode. This mode does not decay before the second measurement location $(\mathrm{x} / \mathrm{c}=0.257)$, in fact, it grows significantly, especially for suction distribution 110 (Fig. 20). This mode reaches a large amplitude (up to $8 \%$ of the freestream velocity) before decaying. In fact, the mode was large enough to cause distortion of the basic state. However, it did not cause premature transition for any of these cases since the mode decayed by the last measurement region $(\mathrm{x} / \mathrm{c}=0.437)$, where the flow was still laminar.

Figure 19. Suction Distributions for Leading Edge Panel 2 cases. 


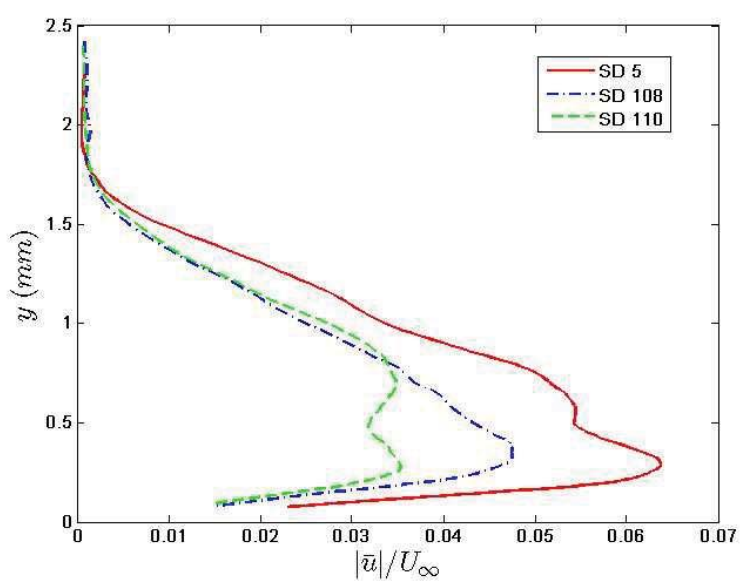

(a) $x / c=0.157$

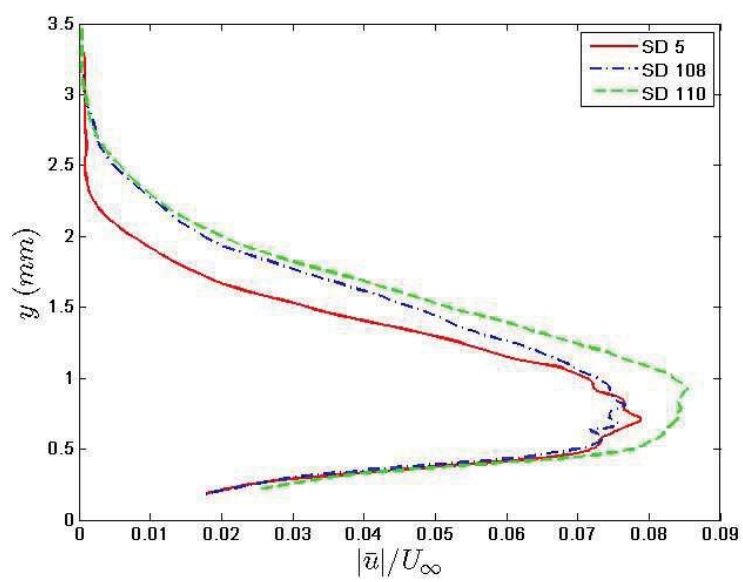

(b) $x / c=0.257$

Figure 20. Stationary crossflow modes $(k=0.25$ to $0.35 \mathrm{~mm}^{-1}$ ) for different suction distributions

The wavelength of the mode with the largest amplitude at the final measurement location $(\mathrm{x} / \mathrm{c}=0.437)$ is between 7 and $10 \mathrm{~mm}$. The evolution of this mode is shown in Fig. 21. This mode is most amplified for Suction Distribution 5 (SD 5) and is about $40 \%$ larger at $\mathrm{x} / \mathrm{c}=0.437$ for the baseline suction distribution than for the other two suction cases.

The results are compared with linear PSE results, which were computed using LASTRAC ${ }^{5}$. For these computations, the surface temperature distribution was specified as in Fig. 12, and the effective suction velocities were specified such that the suction flow rate through each flute matched the experimentally measured flow rates. The $\mathrm{N}$-factors for both modes are plotted in Fig. 22. The experimental $\mathrm{N}$-factors were computed assuming an initial amplitude to match the linear PSE results at the first measurement location $(x / c=0.157)$. For the $4 \mathrm{~mm}$ mode, the experimental

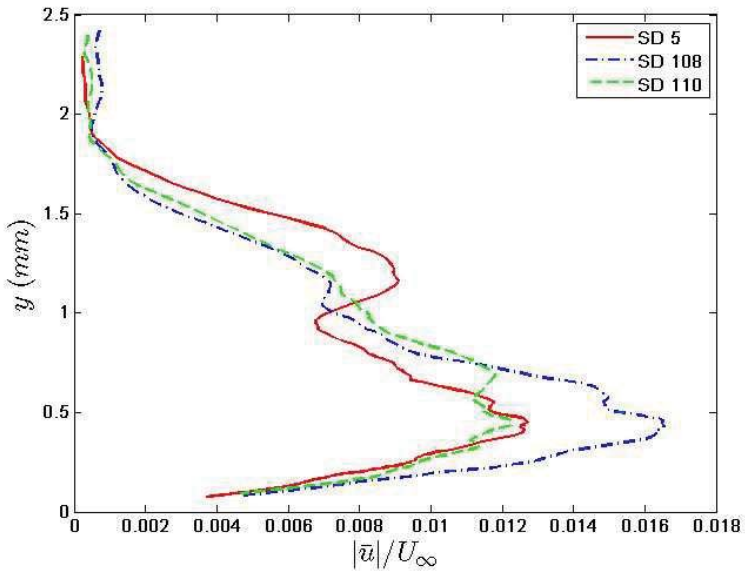

(a) $\mathrm{x} / \mathrm{c}=\mathbf{0 . 1 5 7}$

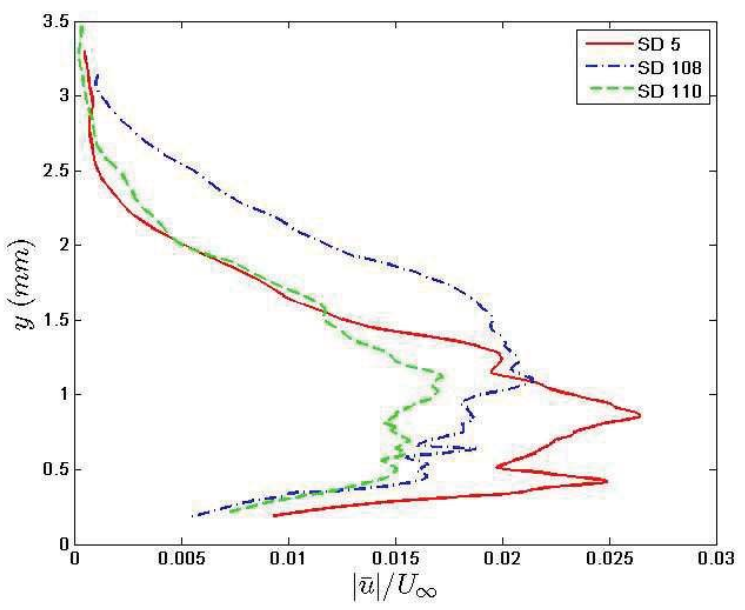

(b) $\mathrm{x} / \mathrm{c}=\mathbf{0 . 2 5 7}$



(c) $\mathrm{x} / \mathrm{c}=\mathbf{0 . 4 3 7}$

Figure 21. Stationary crossflow modes $(k=0.1$ to 0.15 $\mathbf{m m}^{-1}$ ) for different suction distributions 
results at the second location are fairly close for the SD5 and SD108 suction cases. However, the SD110 mode grows much more than predicted by linear PSE. For the $9 \mathrm{~mm}$ mode, the experimental results at the second measurement location show good agreement with the computational results, but the mode is much less amplified than predicted at the last measurement location.

For both modes, the results from the SD5 suction case had the best overall agreement with the predicted $\mathrm{N}$-factor values. However, for both SD108 and SD110, the computational results significantly under-predict the growth of the $4 \mathrm{~mm}$ mode and over-predict the growth of the $9 \mathrm{~mm}$ mode. It is interesting that both of the cases with elevated suction through two flutes show similar trends, particularly the unexpected growth of the 4 mm mode.

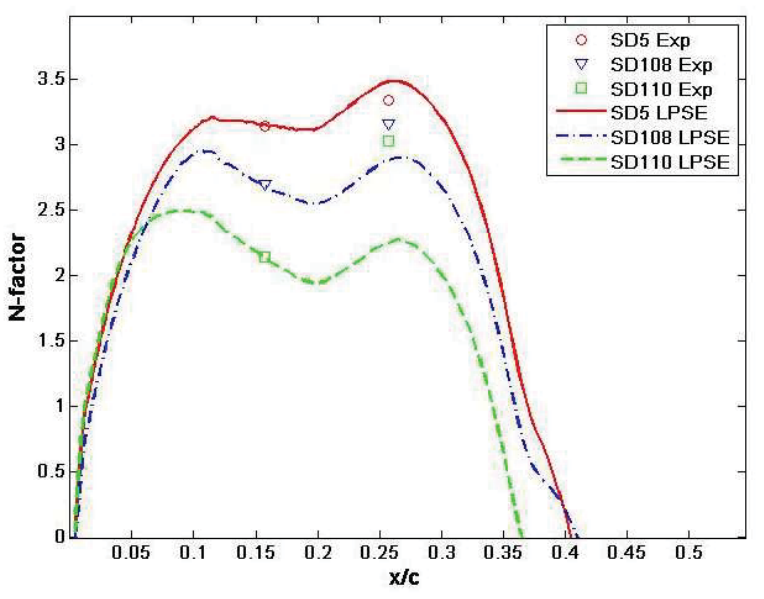

(a) $4 \mathrm{~mm}$ mode $\left(\mathrm{k}=0.25 \mathrm{~mm}^{-1}\right)$

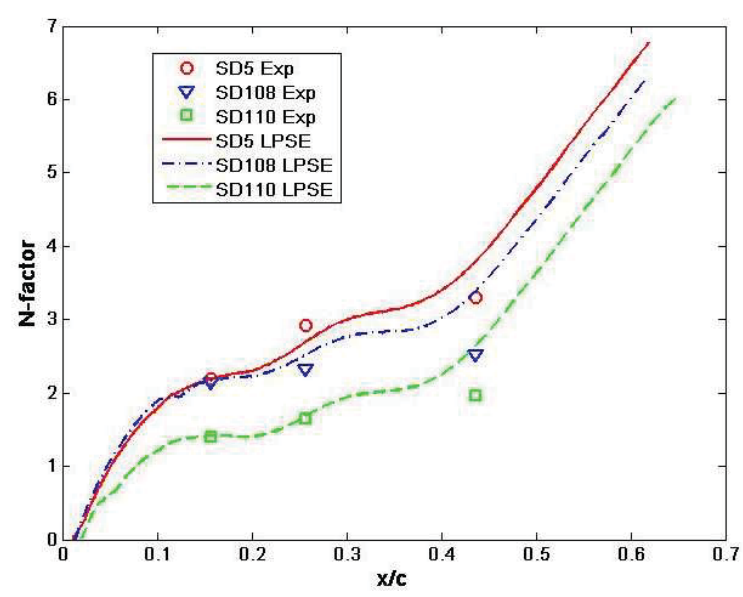

(b) $9 \mathrm{~mm}$ mode $\left(\mathrm{k}=0.11 \mathrm{~mm}^{-1}\right)$

Figure 22. $\quad N$-Factor plots for $4 \mathrm{~mm}$ and $9 \mathrm{~mm}$ modes.

\section{Conclusions}

The NASA/Boeing HLFC Crossflow experiment produced a unique dataset which is useful for increasing our understanding of suction effects on crossflow growth and transition. Several new techniques were developed to aid in the data analysis. The vibration of the probe caused peaks in the spectral density plots of the dynamic hotwire data which could have been mistaken for traveling disturbances. These peaks were identified by looking for qualitative alignment with the velocity gradient of the mean profiles.

The wall location was corrected for effects due to the stationary crossflow distortion of the mean profiles. A low-pass spatial filter was employed to eliminate unrealistic variations in wall location. This resulted in a change in crossflow amplitude of between $10 \%$ and $40 \%$ as well as a change in the mode shape. The smooth wall stationary crossflow mode shapes and the linear-fit wall stationary crossflow mode shapes were compared to linear PSE results. The smooth wall mode shapes showed slightly better agreement with the linear PSE results for most cases.

The results from the hotwire temperature correction suggest that the traveling crossflow disturbance amplitude was highly sensitive to wall temperature. This was investigated by comparing the change in amplitude due to the wall temperature with linear PSE results. The difference in N-factor due to the difference in wall temperature calculated using linear PSE was similar to that seen in the experimental results. This indicates that experimentalists should be careful when designing and carrying out experiments where traveling crossflow is an important factor.

Traveling crossflow at amplitudes up to $1 \%$ of the freestream velocity were present in the data, despite the low turbulence intensity $(0.01-0.02 \%)$ of the 8 -ft TPT at the experimental conditions that were investigated. The mode shapes of the experimental traveling modes match the linear PSE results quite well. The experimental spanwise phase speeds ranged between $8-13 \%$ of the freestream velocity. These results were typically higher than that predicted by linear PSE of around 5\% for the most amplified mode.

Stationary crossflow growth for several different suction distributions was presented. As predicted, the suction hole spacing on the panel excited a crossflow mode at that wavelength. In fact, this mode grew to large amplitudes ( $8 \%$ of the freestream velocity) before decaying. The $\mathrm{N}$-factor results show fairly good agreement between the linear PSE results and the experimental results for two of the suction distributions. However, the crossflow mode for the suction distribution with flutes 5 and 6 at higher suction rates (Suction Distribution 110) grew significantly more than predicted. The results for the larger wavelength modes show good agreement with the linear PSE results before the modes began to exhibit nonlinear effects. 
Now that the results have been refined, efforts have been undertaken to incorporate the post-test porosity measurements of the suction panels with the hotwire data. Non-uniformity of the suction was believed to be present during the experiment due to hole blockage and defects in hole fabrication. Detailed measurements were performed to determine the actual suction distributions that were present during the experiment. The hope is that we will be able to come to some meaningful conclusions regarding the effect of the non-uniformity of the suction on the receptivity and growth of the crossflow modes.

\section{Acknowledgments}

This work was supported by the NASA Subsonic Fixed Wing Project. The authors would like to thank Meelan Choudhari and Craig Streett for their input and support. Cathy Cronin was instrumental in getting the database back up and running, and her help in recovering the data has been much appreciated. We are also indebted to Latunia Melton, who provided us with some key missing information from the test.

\section{References}

${ }^{1}$ Wlezien, R., Eppink, J., and Cronin, C., "Web-based interactive wind tunnel testing - A historical perspective," 27th AIAA Aerodynamic Measurement Technology and Ground Testing Conference, AIAA 2010-4213, Chicago,IL, 2010.

${ }^{2}$ Streett, C. L., "Designing a hybrid laminar-flow control experiment - the CFD-experiment connection," 41st Aerospace Sciences Meeting and Exhibit, AIAA Paper 2003-979, Reno, NV, 2003.

${ }^{3}$ Choudhari, M. M., Li, F., Chang, C. -L.., and Malik, M. R., "NASA/Boeing HLFC experiment: Data analysis with advanced CFD tools," High Technology Report No. HTC-9607, 1996.

${ }^{4}$ High Technology Corporation, "NASA/Boeing HLFC Experiment: Data Analysis," 1996, NAS1-20267.

${ }^{5}$ Chang, C.-L., "Langley stability and transition analysis code (LASTRAC) version 1.2 user manual," 2004, NASA/TM2004-213233.

${ }^{6}$ Fey, U., Engler, R.H., Egami, Y., Iijima, Y., Asai, K., Jansen, U., and Quest, J., "Transition detection by temperature sensitive paint at cryogenic temperatures in the European Transonic Wind Tunnel (ETW)," $20^{\text {th }}$ International Congress on Instrumentation in Aerospace Simulation Facilities ICIASF, Gottingen, Germany, 2003.

${ }^{7}$ Deyhle, H., Hohler, G., and Bippes, H., "Experimental investigation of instability wave propagation in a three-dimensional boundary-layer flow," AIAA Journal. Vol. 31, No. 4, April 1993, pp. 637-645.

${ }^{8}$ White, E. and Saric, W. S., "Secondary instability of crossflow vortices," Journal of Fluid Mechanics, 2005, Vol. 525, pp. 275-308.

${ }^{9}$ Dagenhart, J. R. and Saric, W. S., "Crossflow stability and transition experiments in swept-wing flow," NASA/TP-1999209344, 1999.

${ }^{10}$ Wlezien, R.S., Spencer, S.A., and Grubb, J.P., "Comparison of flow quality in subsonic pressure tunnels," $18^{\text {th }} A I A A$ Aerospace Ground Testing Conference, AIAA 1994-2503, Colorado Springs, CO, 1994.

${ }^{11}$ Takagi, S. and Itoh, N., "Observation of Traveling Waves in the Three-Dimensional Boundary Layers along a Yawed Cylinder," Fluid Dynamics Research, Vol. 14, No. 4, 1994, pp. 167-189.

${ }^{12}$ Pfenninger, W., Gross, L., and Bacon, J. W., "Experiments on a 30 degree swept 12\%-thick symmetrical laminar suction wing in the 5-ft. by 7-ft. Michigan tunnel," NAI-57-317 (BLC-93), Northrop Aircraft, Inc., 1957.

${ }^{13}$ Maddalon, D. V., Collier, F. S., Jr., Montoya, L. C., and Putnam, R. J., “Transition flight experiments on a swept wing with suction," Laminar-turbulent transition, Springer-Verlag, 1990, pp. 53-64.

${ }^{14}$ Goldsmith, J., "Critical suction quantities and pumping losses associated with laminar boundary layer suction through rows of closely-spaced holes,” Northrup Aircraft Inc. Rep. NAI-55-287 (BLC-72), 1955. 